\title{
Vesicoureteral reflux: From prophylaxis to surgery
}

\author{
Anne-Sophie Blais, MD;1 Stéphane Bolduc, MD;1,2 Katherine Moore, $M D^{1}$ \\ 'Division of Urology, CHU de Québec-Université Laval, Quebec City, QC, Canada; ${ }^{2}$ RCHU de Québec-Université Laval, Quebec City, QC, Canada and Axe Médecine Régénératrice
}

Cite as: Can Urol Assoc J 2017;11 (1-2Suppl1):S13-8. hitp://dx.doi.org/10.5489/cuaj.4342

See related commentary on page S19.

\section{Abstract}

Vesicoureteral reflux (VUR) is one of the most common pathologies encountered in pediatric urology. Better understanding of the evolution of VUR and new endoscopic surgical techniques in the last decades have led to major changes in the management of this pathology. However, the treatment algorithm remains complex and is composed of a wide variety of options, from active surveillance to surgical treatment. Herein, we propose to review treatment options for VUR in order to help clinicians make the right treatment decision for the right patient.

\section{Introduction}

Vesicoureteral reflux (VUR) is the retrograde flow of urine from the bladder to the upper urinary tract. It is the end result of several anomalies related to the functional integrity of the ureter, the dynamics of the bladder, and the anatomic composition of the ureterovesical junction (UVJ). Clinical presentation is variable, but most patients are either asymptomatic (hydronephrosis) or present with pyelonephritis. Treatment options can be divided into medical and surgical management. Conservative therapy is based on two principal approaches: active surveillance and antibiotic prophylaxis. Surgical management of VUR can be done with either endoscopic treatment or open/laparoscopic/robotic surgical techniques. However, many aspects related to the identification, followup, and management of VUR are still controversial. We propose a review of the different treatment options available for VUR.

\section{Active surveillance}

Clinical management of VUR is complex and should be individualized. Treatment does not always confer any true clinical benefit in selected children. The main health con- cern in patients with VUR is the occurrence of febrile urinary tract infection (UTI) or pyelonephritis, which may lead to renal scarring, hypertension, and renal insufficiency. On the other hand, VUR has a high rate of spontaneous resolution, especially in young patients and low-grade VUR. Keeping this in mind, the goals of treatment are to minimize overtreatment in patients with low risk of UTI and to prevent renal scarring. Many studies and guidelines have tried to better identify the minority of children in whom VUR is significant enough to warrant early treatment. However, as stated earlier, management of VUR remains complex and individualized. When a patient and his or her parents choose to attempt active surveillance, it is essential to counsel the family about the management of UTI risk factors.

Bladder and bowel training is the mainstay of conservative treatment. In 2010, the American Urological Association (AUA) developed guidelines particularly addressing the association of bladder and bowel dysfunction (BBD) and VUR. ${ }^{1}$ Their meta-analysis showed a lower rate of VUR selfresolution, an increased risk of UTI, and a decreased rate of successful correction of reflux after surgery in children with non-treated BBD. Children experiencing bladder dysfunction should go through bladder retraining in order to ensure regular and complete emptying of the bladder and low-pressure voiding. A voiding routine can help remind the child to void regularly. Position adopted by the children (feet well-positioned) during voiding is also critical to permit pelvic floor muscle relaxation. Moreover, biofeedback may be used in the pediatric population to gain better awareness and voluntary control over pelvic floor muscles. ${ }^{2}$ Antimuscarinic therapy may also be needed to stabilize overactive bladder if associated symptoms are clearly identified.

Constipation is a symptom of BBD that is common in patients with VUR and increases the risk of $\mathrm{UTI}^{2}{ }^{2}$ thereby, it should be addressed aggressively. Maintaining a good level of hydration prevents constipation and contributes to the prevention of UTI, as it stimulates more frequent micturition. A diet rich in fibers may help in obtaining soft stools. Persistent constipation should be treated with laxatives. It is the authors' preference to use polyethylene glycol when constipation is not resolved with appropriate diet and hydration. 
Circumcision may also be part of the conservative management in children younger than one year of age, as it decreases UTI risks. According to the AUA: "The established reduction in UTI, however, coupled with the risk of UTI in infants with VUR, prompted the panel to consider circumcision an option in the management of the infant boy with VUR."1

\section{Antibiotic prophylaxis}

Traditionally, continuous antibiotic prophylaxis (CAP) was the first-line therapy for most patients in order to prevent febrile UTIs and renal scarring. A recent shift to early surgical treatment has been seen due to the emerging success of endoscopic injection therapy. However, even if VUR can be corrected successfully with minimally invasive therapy, reflux correction is not mandatory in all patients. Eighty percent of low-grade and $50 \%$ of Grade III reflux will resolve spontaneously without treatment. ${ }^{3}$ Daily low dose of CAP is used in order to maintain sterile urine and prevent renal scarring occurring in the setting of febrile UTI. It may also offer more time for reflux to resolve spontaneously, avoiding the morbidity associated with surgical treatment. Recent studies showed conflicting evidence on the effectiveness of CAP for the prevention of UTI. In the RIVUR study, prophylaxis reduced the risk of febrile or symptomatic UTI recurrence by $50 \%$ compared to placebo. ${ }^{4}$ On the other hand, some studies demonstrated lack of effectiveness of CAP to prevent pyelonephritis recurrences, renal scarring, and UTI. ${ }^{5,6}$ Still, the AUA and the European Association of Urology (EAU) guidelines consider CAP a reasonable option for initial therapy for the majority of children with VUR. ${ }^{1,7}$

While prescribing CAP to pediatric patients, a few considerations must be taken into account. Sulfamethoxazole (SMX) and nitrofurantoin should not be prescribed in children younger than two months of age because of their hepatic immaturity. Antibiotic dosage must consistently be adjusted to children's growth and weight. Moreover, CAP should be given at night in the toilet-trained children, allowing longer concentrations of antibiotic in urine. CAP is usually stopped as soon as VUR resolution is documented. In our practice, CAP is also reassessed after acquisition of toilet-training because it represents a critical period for development of UTI. UTIs are less likely to occur with appropriate bowel and bladder habits. It is also believed that after the age of five, kidneys become less susceptible to scarring due to infection. ${ }^{3}$ We now see a trend toward early discontinuation of CAP in the literature and promotion of active surveillance. ${ }^{8}$ The Canadian Pediatric Society no longer routinely recommends CAP for Grades I-III reflux and suggests reassessing CAP every 3-6 months to decrease occurrence of adverse events and microbial resistance.

\section{Surgical treatment}

\section{Indications}

As with CAP, the indications for surgical treatment of VUR remain controversial. The goal in selecting patients for surgical treatment is to identify the ones who are unlikely to spontaneously resolve VUR and the ones at higher risk for pyelonephritis and renal scarring. Patients' age, gender, history of UTIs, renal status, grade of VUR, social status, patients' preferences, and the probability and timing of spontaneous resolution of VUR may help identify high-risk patients. Surgical repair is commonly offered to patients who suffer from breakthrough UTIs despite the use of CAP. Patients presenting with persistent high-grade reflux, particularly if bilateral, or new renal scars are also candidates for surgical treatment. Moreover, surgery is a reasonable option in patients with restricted access to healthcare services or poor compliance to non-surgical treatment. A subset of girls with persistent VUR after puberty will undergo corrective surgery, as reflux in adult females carries an increased propensity for maternal and fetal morbidity during pregnancy, especially with presence of scars/dysplasia..$^{9}$ Finally, patient and family's preferences may affect the decision to undergo a surgical treatment after discussion and informed consent.

\section{Endoscopic injection}

The role of endoscopic injection therapy is rapidly evolving since the techniques and the bulking agents are constantly improving. The goal of endoscopic injection of VUR is to create a solid support behind the intravesical ureter without impeding the normal flow of urine from the kidneys to the bladder. This support allows elongation of the intramural tunnel of the ureter. Indications for endoscopic injection therapy are now applied to a wider range of patients, extending the indications to more complexes cases. Highrisk groups like Grade IV VUR, duplicated systems, or previous treatment failures are now considered eligible for endoscopic management, but they may have a lower expected success rate. ${ }^{10}$ Before attempting endoscopic injection, it is important to make sure patients are compliant to behavioural therapy, as voiding dysfunction may increase the risk of treatment failure and recurrent UTI. ${ }^{11}$ Endoscopic treatment of VUR has many advantages: high success rates, day surgery, absence of scar and short postoperative recovery, low risk of significant postoperative complications, safety of injectable materials, and low cost. Endoscopic injection of VUR is now considered a first-line surgical treatment for lowgrade reflux despite the fact that it may not be as effective as ureteral reimplantation, especially for high-grade VUR, and that long-term efficacy is still unknown. Finally, endoscopic 
injection has been shown to be effective in adults with VUR It remains a good surgical option for patients with VUR and breakthrough UTI after puberty. ${ }^{12}$

\section{Injection technique}

Endoscopic injection of VUR is usually performed as a day surgery. The cystoscopy is first performed with the patient under general anesthesia and lithotomy position. The injection is done with a needle that is placed through the operating channel of the cystoscope. The material needed (needle, injecting syringe) may differ from the chosen bulking agent and its manufacturer. The three most popular techniques described in the literature are the subureteral Teflon injection (STING), the hydrodistention technique (HIT), and the double hydrodistention technique (double-HIT). STING technique was the first to be described. The technique consists of the injection of bulking agent, regardless of the agent, into the detrusor muscle immediately beneath the ureteral orifice at the six o'clock position. With the HIT, the ureter is distended with irrigation fluid from the cystoscope and the injection is made within the ureteral orifice, beneath the mucosa. The double-HIT is similar, but two injections are performed. A first injection is performed more proximally, within the ureteral tunnel, and a second injection more distally, just under the ureteral orifice.

Many series tried to determine the optimal technique, however, the results are inconsistent. Regardless of the technique used, it is recommended to avoid multiple punctures and to wait 20-30 seconds with the needle in place after the injection to avoid agent leakage. The appearance of the mound is predictive of the volume of bulking agent to inject. The preferred appearance is a volcano shape with adequate coaptation of the ureteral orifice and correction of the previous hydrodistention. At the end of the procedure, the mound is inspected and the bladder is emptied. After the surgery, patients can immediately return to their normal activities. Followup usually includes an ultrasound to rule out de novo hydronephrosis/obstruction. A voiding cystourethrography is performed in select cases.

\section{Bulking agents}

Several bulking agents have been described for the endoscopic treatment of VUR. Teflon was the first agent used, with many series showing high success rates; one study even showed a higher overall success over cross-linked bovine dermal collagen, Deflux, and autologous blood. ${ }^{13}$ However, Teflon has never obtained U.S. Food and Drug Administration (FDA) approval because of concerns of particle migration.

Cross-linked bovine collagen has been the subject of many trials, revealing inconsistent success rates and high relapse rates. ${ }^{14,15}$ The variable degree of ingrowth of native fibroblasts, the shrinkage of the bulking agent, and the reported immunological reactions after injection of collagen are reasons why cross-linked bovine collagen has been abandoned by most centres.

Injection of autologous material is appealing, as they behave as free grafts with the absence of foreign materials. Blood, chondrocytes, fat, and myoblasts were studied. Fat and chondrocytes showed high rates of long-term VUR recurrence. ${ }^{16-18}$ On the other hand, blood has not been largely studied and the experimental use of myoblasts in pigs failed. ${ }^{19}$

Calcium hydroxyapatite, a synthetic agent with identical chemical composition to teeth and bone, was investigated in animals and humans. Only few studies are available in the literature and reported success rates are widely variable. ${ }^{20,21}$ Further studies with larger cohorts and longer followup are needed.

Polydimethylsiloxane (PDMS, Macroplastique ${ }^{\circledR}$ ) and dextranomer/hyaluronic acid (Dx/HA, Deflux ${ }^{\circledR}$ ) are two popular bulking agents. PDMS has gained popularity in the treatment of VUR because of its low risk of migration due to its large particle size, composition, texture, and the absence of shrinkage. It was FDA-approved for transurethral injection of stress urinary incontinence in 2006, but used for more than 25 years in Europe and since the late 90s in Canada. Many urologists have recorded their success rate with PDMS. Herz et al have shown an overall success rate of $81 \%$ after a single injection at an average followup of 18 months. ${ }^{22}$ A review reported an overall success rate at one year, two years', and nine years' followup of $86-93 \%, 80-92 \%$, and $77-100 \%$ respectively. ${ }^{23}$ However, a specific gun for the injection of PDMS is needed to ensure high pressure during injection and it is technically more difficult to inject.

On the other hand, Dx/HA is the only FDA-approved bulking agent for endoscopic injection of VUR in children. Depending on the reflux grade, the overall success rate reported in the literature ranges from $68-92 \% .{ }^{24} \mathrm{~A}$ systematic review has shown an overall success rate per ureter of $77 \%$ at three months, while a German study noted a success rate of $81.5 \%$ at three months. ${ }^{25,26}$ Many groups have compared the results obtained with a single injection of PDMS vs. Dx/HA copolymer for VUR endoscopic treatment. The results are variable, ranging from no difference to a better success rate with PDMS. ${ }^{27}$

Two agents have been recently introduced for the endoscopic injection of VUR: polyacrylate-polyalcohol copolymer (PPC, Vantris ${ }^{\circledR}$ ) and polyacrylamide hydrogel (PAHG, Bulkamid ${ }^{\circledR}$ ). A multicentre survey showed a success rate of $93.8 \%$ after a single injection of PPC. ${ }^{28}$ Patients were monitored with ultrasound and voiding cystourethrogram. Most renal refluxing units had higher-grade VUR $(55.8 \%$ Grade III and $19.5 \%$ Grade IV) and most patients (60\%) 
were followed for more than two years. Moreover, PPC and Dx/HA have been compared in many trials. ${ }^{29-31}$ Endoscopic injection of PPC resulted in higher success rates. However, concerns have been raised with PPC regarding a high rate of ureteral obstruction. Finally, only few published series are available on PAHG. ${ }^{32-34}$ The success rate with this bulking agent appears promising, but more trials are needed prior to extensive use.

\section{Complications}

The rate of major complications with VUR endoscopic injection is low. Hematuria can occur following injection. It is usually mild. When it happens intraoperatively, it is recommended to empty the bladder and to gently apply the tip of the cystoscope at the bleeding site until the bleeding stops. UTI may also occur. Most patients already have a history of UTI and are at high risk of recurrence. It is of great importance to obtain a urine culture prior to surgery and to treat bacteriuria.

De novo contralateral VUR can also occur after surgery. The rate of contralateral low-grade VUR is reported at $4.5-7 \% .^{35}$

Obstruction is one of the most common complications reported in the literature. It may manifest by renal colic, de novo hydroureteronephrosis, UTI, or lower urinary tract symptoms. Many treatments have been described to treat ureteral obstruction: ureteral stenting, percutaneous nephrostomy, ureteral reimplantation, balloon dilatation, and nephroureterectomy. However, it is often transient.

Finally, the implanted agent can become calcified. This can lead to hematuria, intermittent back pain, necrosis of the bladder mucosa, erosion of the bulking agent, and wrong diagnosis of distal ureteral lithiasis. Long-term impact of this condition are still misunderstood, however, several nondiagnostic ureteroscopies are expected to be performed despite positive imaging if adult urologists are not aware of this possible complication or of the fact that the patient underwent VUR injection at a younger age.

\section{Ureteral reimplantation}

Multiple surgical techniques have been described following the initial report of Hutch. Landmark refinements of Hutch's surgery include the transvesical advancement technique (Politano-Leadbetter), ${ }^{36}$ Paquin,${ }^{37}$ the extravesical Lich-Grégoir, ${ }^{38,39}$ the distal tunnel advancement (GlennAnderson), ${ }^{40}$ and the transtrigonal technique (Cohen) ${ }^{41}$ These procedures can be classified on the base of the approach use (intravesical, extravesical, or combined) and the position of the submucosal tunnel (suprahiatal or infrahiatal). The best procedure remains individualized according to patient's condition and surgeon's experience/preference.
Regardless of the type of procedure, many surgical principles may be followed to obtain a good success rate. Successful surgery includes creation of a sealed tensionfree ureteral anastomosis, preservation of the delicate blood supply to the distal ureter, creation of a submucosal tunnel length five times longer than the diameter of the ureter over a firm backing, delicate handling of the bladder, and an adequate anastomosis preventing stenosis, twisting, and wrong angulation of the ureter. Prophylactic antibiotics are generally administered before the induction of general anesthesia. Some surgeons perform cystoscopy at the beginning of the procedure. Cystoscopy can be helpful in identifying subtle anomalies such as diverticula and small ureterocele. The contralateral UVJ can also be examined with hydrodistention to detect occult VUR. After cystoscopy, the patient is positioned supine. A Pfannenstiel incision is usually used to gain access to the bladder. Then, the steps differ according to the technique used.

The Politano-Leadbetter and Paquin techniques are classified as suprahiatal repair. The goal of the Politano-Leadbetter technique is to create a new hiatus superior to the original hiatus by an intravesical approach. The tunnel is directed toward the trigone, medial to the original hiatus. This procedure allows the creation of a long tunnel, which is optimal in case of high-grade reflux or large ureter. Paquin's reimplantation combines the extravesical and intravesical dissection. The new hiatus is created from outside the bladder. The combined approach and the possibility to create a long tunnel make it a suitable approach for dilated ureters, complex cases, and previously failed reimplantations.

The techniques described by Glenn-Anderson and Cohen are classified as infrahiatal and intravesical. In the GlennAnderson procedure, the ureter is advanced distally toward the bladder neck. However, because the distance between the hiatus and the bladder neck is short, it limits the length of the tunnel. Cohen's technique overcomes this limitation. The tunnel is directed across the trigone toward the contralateral bladder wall.

Finally, the extravesical approach has the advantage of minimizing hematuria and bladder spasms frequently seen with the intravesical approaches. The main concern with the extravesical approach is the transient voiding inefficiency that may be seen in children with bilateral reimplantation. ${ }^{42}$ The hypothesis for this dysfunction is that the disruption of nerves during the detrusorotomy could alter detrusor contractility. Transient voiding dysfunction has also been described with cases of more extensive dissection by intravesical approaches

Transperitoneal laparoscopic extravesical and intravesical ureteral reimplantations have been reported by several groups. Laparoscopic extravesical techniques seem to be especially beneficial in the adolescent and adult populations. However, transvesical laparoscopic approach is more 
challenging, with long operative time, small working space, long learning curve, and difficulty in obtaining secure closure at the bladder wall level at the time of port removal. ${ }^{43}$ Robot-assisted laparoscopic ureteral reimplantation (extra and intravesical) has been more and more adopted in North America. However, a wide range of success rates and many variations in the surgical technique are reported. More studies are needed to define the costs and benefits of robotassisted laparoscopic ureteral reimplantation over the gold standard open approach, which can now also be performed in a "mini reimplantation" fashion.

\section{Complications}

As with endoscopic injection, UTI, hematuria, contralateral VUR, and obstruction have been reported with ureteral reimplantation. Obstruction occurs at an estimated rate of $1 \% .^{44}$ Initial management usually includes derivation with an indwelling catheter or percutaneous nephrostomy. Early transient obstruction is frequently due to edema of the UVJ. On the other hand, persistent obstruction is often due to stenosis or kinking of the ureter and may need a repeat reimplantation. The rate of de novo contralateral reflux is $5-18 \%$ in open series. ${ }^{35}$ This reflux is usually low-grade and may resolve spontaneously. Transient voiding dysfunction, as stated earlier, may also occur after surgery. It usually resolves spontaneously within 1-2 weeks. Insertion of a Foley catheter or intermittent catheterization may be required until resolution of urinary retention.

\section{Conclusion}

VUR is a complex pathology that is still not fully understood. Observation, continuous antibiotic prophylaxis, endoscopic injection of a bulking agent, and ureteral reimplantation are all valuable options to treat this condition. The goals of treatment are to prevent renal scarring while avoiding unnecessary invasive procedures. In this context, individualized treatment is of great importance. The future may yield more knowledge on the natural history of VUR and improvements in treatment procedures.

Competing interests: Dr. Bolduc has received grant funding for clinical trials from Astellas Pharma and Pfizer Canada; has been a principal investigator for clinical projects associated with Astellas Pharma and Pfizer Canada on overactive bladder; and was the recipient of the Canadian Urological Association Scholarship Fund (CUASF) and CUA-Astellas research grants. Dr. Moore has been a clinical investigator for projects associated with Astellas Pharma and Pfizer Canada on overactive bladder and Ipsen for neurogenic bladder; is a speaker for Astellas, Ferring, and Pfizer; and has served on advisory boards for Astellas and Pfizer. Dr. Blais reports no competing personal or financial interests.

This paper has been peer-reviewed.

\section{References}

1. American Urological Association pediatric vesicoureteral reflux clinical guidelines panel. Management and screening of primary vesicoureteral reflux in children: AUA guideline. Baltimore: American Urological Association, Inc., 2010.

2. Elder JS, Diaz M. Vesicoureteral reflux - the role of bladder and bowel dysfunction. Nat Rev Urol 2013;10:640-8. htrps://doi.org/10.1038/nrurol.2013.221

3. Wein AJ, Kavoussi LR, Partin AW, et al. Campbell-Walsh Urology. 11th Edition. 2016.

4. Hoberman A, Greenfield SP, Mattoo TK, et al. Antimicrobial prophylaxis for children with vesicoureteral reflux. N Engl J Med 2014;370:2367-76. https://doi.org/10.1056/NEJMoal 401811

5. Pennesi, $M$, Travan, L, Peratoner L, et al. Is antibiotic prophylaxis in children with vesicoureteral reflux effective in preventing pyelonephritis and renal scars? A randomized, controlled trial. Pediatrics 2008;121:148994. https://doi.org/10.1542/peds.2007-2652

6. Conway PH, Cnaan A, Zaoutis T, et al. Recurrent urinary tract infections in children. JAMA 2007;298:17986. https://doi.org/10.1001/jama.298.2.179

7. Tekgül S, Riedmiller $H$, Hoebeke $P$, et al. EAU guidelines on vesicoureteral reflux in children. Eur Urol 2012;62:534-42. https://doi.org/10.1016/i.eururo.2012.05.059

8. Moriya K, Mitsui T, Kitta T, et al. Discontinuation of antibiotic prophylaxis in patients with persistent primary vesicoureteral reflux initially detected during infancy: Outcome analysis and risk factors for febrile urinary tract infection. J Urol 2015;193:637-42. htrps://doi.org/10.1016/i.juro.2014.08.007

9. Hollowell JG. Outcome of pregnancy in women with a history of vesicoureteric reflux. BJU Int 2008;102:780-4. https://doi.org/10.1111/j.1464-410X.2008.07671.x

10. Perez-Brayfield M, Kirsch AJ, Hensle TW, et al. Endoscopic treatment with dextranomer hyaluronic acid for complex cases of vescioureteral reflux. J Urol 2004;172:1614-6. https://doi.org/10.1097/01. ju. 0000139013.00908.1c

11. Diamond D, Mattoo TK. Endoscopic treatment of primary vesicoureteral reflux. N Engl J Med 2012;366:1218-26. https://doi.org/10.1056/NEJMct1 108922

12. Moore K, Bolduc $S$. Treatment of vesicoureteral reflux in adults with endoscopic injection. Urology 2011;77:1284-7. https://doi.org/10.1016/i.urology.2010.12.080

13. Sugiyama $\mathrm{T}$, Hanai $\mathrm{T}$, Hashimoto $\mathrm{K}$, et al. Long-term outcome of the endoscopic correction of vesicoureteric reflux: A comparison of injected substances. BJU Int 2004;94:381-3. https://doi.org/10.1111/i.1464410X.2004.04966.X

14. Frey $P$, Berger $D$, Jenny $P$, et al. Subureteral collagen injection for the endoscopic treatment of vesicoureteral reflux in children. Followup study of 97 treated ureters and histological analysis of collagen implants. J Urol 1992;148:718-23.

15. Stredele RJF, Dietz HG, Stehr M. Long-term results of endoscopic treatment of vesicoureteral reflux in children: Comparison of different bulking agents. Pediatr Urol 2011;12:1-6.

16. Palma PCR, Ferreira $\mathrm{U}$, Ikari 0 , et al. Subureteric lipoinjection for vesicoureteral reflux in renal transplant candidates. Urology 1994;43:174-7. https://doi.org/10.1016/0090-4295(94)90039-6

17. Olson ME, Morck DW, Ceri H, et al. Evaluation of autologous fat implantation in the rat urinary bladder submucosa. Urology 1998;52:915-9. https://doi.org/10.1016/S0090-4295(98)00338-0

18. Caldamone AA, Diamond DA. Long-term results of the endoscopic correction of vesicoureteral reflux in children using autologous chondrocytes. J Urol 2001;165:2224-7. https://doi.org/10.1016/S0022$5347(05) 66170-8$

19. Mitterberger $M$, Marksteiner $R$, Schwaiger $W$, et al. Can autologous myoblasts be used as a potential bulking agent? BJU Int 2008;102:1731-6. https://doi.org/10.1111/i.1464-410X.2008.07892.x

20. Tarcan T, Tinay I, Temiz Y. Long-term results of endoscopic treatment of vesicoureteral reflux with the subureteric injection of calcium hydroxyapatite. Int Urol Nephrol 2007;39:1011-4. https://doi.org/10.1007/s1 1255-007-9222-x

21. Ngo TC, Wong IYZ, Kennedy WA. A comparison of calcium hydroxyapatite and dextranomer/hyaluronic acid for the endoscopic treatment of vesicoureteral reflux. Adv Urol 2013;2013: 263602. https://doi.org/10.1155/2013/263602

22. Herz D, Hafez A, Bagli D, et al. Efficacy of endoscopic subureteral polydimethylsiloxane injection for treatment of vesicoureteral reflux in children: A North American clinical report. J Urol 2001;166:1880-6. https://doi.org/10.1016/S0022-5347(05)65712-6

23. Aubert $D$. Vesicoureteric reflux treatment by implant of polydimethylsiloxane (Macroplastique): Review of the litterature. Prog Urol 2010;20:251-9. hitps://doi.org/10.1016/i.purol.2009.10.017

24. Chertin B, Kocherov $S$, Chertin $L$, et al. Endoscopic bulking materials for the treatment of vesicoureteral reflux: A review of our 20 years of experience and review of the literature. Adv Urol 2011;2011:309626. https://doi.org/10.1155/2011/309626

25. Routh JC, Inman BA, Reinberg Y. Dextranomer/hyaluronic acid for pediatric vesicoureteral reflux: Systematic review. Pediatrics 2010;125:1010-9. https://doi.org/10.1542/peds.2009-2225 
Blais et al.

26. Stredele RJF, Dietz HG, Stehr M. Long-term results of endoscopic treatment of vesicoureteral reflux in children: Comparison of different bulking agents. J Pediatr Urol 2011;12:1-6.

27. Moore K, Bolduc S; Prospective, randomized study of endoscopic injection for pediatric VUR: Macroplastique vs. deflux. J Urol 2014;192:1794-1800. https://doi.org/10.1016/i.juro.2014.05.116

28. Stanislav K, Ibrahim U, Sergey N. Multicentre survey of endoscopic treatment of vesicoureteral reflux using polyacrylate-polyalcohol bulking copolymer (Vantris). Urology 2014;84:689-93. hitps://doi.org/10.1016/i.urology.2014.04.033

29. Warchol S, Krzemien G, Szmigielska A, et al. Comparison of results of endoscopic correction of vesicoureteral reflux in children using two bulking substances: Dextranomer/hyaluronic acid copolymer (Deflux) vs. polyacrylate-polyalcohol copolymer (Vantris). J Pediatr Urol 2016;12:256. https://doi.org/10.1016/i. ipurol.2016.04.006

30. Kocaoglu C. Endoscopic treatment of Grades IV and V vesicoureteral reflux with two bulking substances: Dextranomer hyaluronic acid copolymer vs. polyacrylate polyalcohol copolymer in children. J Pediatr Surg 2016:51:1711-5. https://doi.org/10.1016/i.pedsurg.2016.03.013

31. Karakus SC, User R, Kilic BD. The comparison of dextranomer/hyaluronic acid and polyacrylate-polyatcohol copolymers in endoscopic treatment of vesicoureteral reflux. J Pediatr Surg 2016; 1:1496-500. https://doi.org/10.1016/i.jpedsurg.2016.02.092

32. Cloutier J, Blais AS, Moore K, et al. Prospective study using a new bulking agent for the treatment of vesicoureteral reflux: Polyacrylamide hydrogel. J Urol 2013;190:1034-7. https://doi.org/10.1016/i. juro.2013.03.071

33. Blais AS, Morin F, Cloutier J, et al. Efficacy of dextranomer hyaluronic acid and polyacrylamide hydrogel in endoscopic treatment of vesicoureteral relfux: A comparative study. Can Urol Assoc J 2015;9:202-6. https://doi.org/10.5489/cuaj.2964
34. Ramsay S, Blais AS, Morin F, et al. Polyacrylamide hydrogel as a bulking agent for the endoscopic treatment of vesicoureteral reflux: Long-term results and safety. J Urol 2016; [Epub ahead of print]. https://doi.org/10.1016/i.juro.2016.08.093

35. Lorenzo AJ, Khoury AE. Endoscopic treatment of reflux: Management pros and cons. Curr Opin Urol 2006;16:299-304. https://doi.org/10.1097/01.mou.0000232054.12461.5f

36. Politano VA, Leadbetter WF. An operative technique for the correction of vesicoureteral reflux. J Urol 1958;79:932-41.

37. Paquin AJ Jr. Ureterovesical anastomosis. J Urol 1959;82:573-83.

38. Lich R Jr, Howerton LW, Davis LA. Ureteral reflux, its significance and correction. South Med J 1962:55:6335. https://doi.org/10.1097/00007611-196206000-00020

39. Gregoir W. The surgical treatment of congenital vesicoureteral reflux. Acta Chir Belg 1964;63:431-9.

40. Glenn JF, Anderson EE. Distal tunnel ureteral reimplantation. J Urol 1967;97:623-6.

41. Cohen SJ. The Cohen reimplantation technique. Birth Defects Orig Artic Ser 1977;13:391-5.

42. Fung LCT, McLorie GA, Jain U, et al. Voiding efficiency after ureteral reimplantation: A comparison of extravesical and intravesical techniques. J Urol 1995;153:1972-5. https://doi.org/10.1016/S0022$5347(01) 67381-6$

43. Hong $\mathrm{CH}$, Kim JH, Jung HJ, et al. Single-surgeon experience with transvesicoscopic ureteral reimplantation in children. Urology 2011;77:1465-9. https://doi.org/10.1016/j.urology.2010.11.023

44. Steffens J, Langen PH, Haben B, et al. Politano-leadbetter ureteroneocystostomy: A 30-year experience. Urol Int 2000;65:9-14. https://doi.org/10.1159/000064827

Correspondence: Dr. Katherine Moore, CHU de Québec-Université Laval (CHUL), Quebec City, QC, Canada; katherine.moore.1@ulaval.ca 